\author{
MITSUBISHI ELECTRIC RESEARCH LABORATORIES \\ http://www.merl.com
}

\title{
Effect of far scatterer clusters in MIMO outdoor channel models
}

\author{
Molisch, A.
}

TR2003-38 May 2003

\begin{abstract}
We consider the clustering structure of wireless MIMO channels, and the impact that multiple clusters have on the performance of MIMO systems. We show that multiple clusters frequently occur in urban environments. We then proceed to demonstrate that a channel with multiple scatterer clusters behaves differently than a channel with one cluster and the same rms angular spread. The differences are quantified in terms of correlation coefficients, information-theoretic capacity, and interference-suppression capabilities. This demonstrates the necessity of correctly taking into account the clustering structure.
\end{abstract}

IEEE, Vehicular Technology Conference, Spring 2003, Jeju, Korea

\footnotetext{
This work may not be copied or reproduced in whole or in part for any commercial purpose. Permission to copy in whole or in part without payment of fee is granted for nonprofit educational and research purposes provided that all such whole or partial copies include the following: a notice that such copying is by permission of Mitsubishi Electric Research Laboratories, Inc.; an acknowledgment of the authors and individual contributions to the work; and all applicable portions of the copyright notice. Copying, reproduction, or republishing for any other purpose shall require a license with payment of fee to Mitsubishi Electric Research Laboratories, Inc. All rights reserved.
} 



\author{
MERL - A MITSUBISHI ELECTRIC RESEARCH LABORATORY \\ http://www.merl.com
}

\title{
Effect of far scatterer clusters in MIMO outdoor channel models
}

\author{
Andreas F. Molisch
}

TR-2003-38 May 2003

\begin{abstract}
We consider the clustering structure of wireless MIMO channels, and the impact that multiple clusters have on the performance of MIMO systems. We show that multiple clusters frequently occur in urban environments. We then proceed to demonstrate that a channel with multiple scatterer clusters behaves differently than a channel with one cluster and the same rms angular spread. The differences are quantified in terms of correlation coefficients, information-theoretic capacity, and interference-suppression capabilities. This demonstrates the necessity of correctly taking into account the clustering structure.
\end{abstract}

This work may not be copied or reproduced in whole or in part for any commercial purpose. Permission to copy in whole or in part without payment of fee is granted for nonprofit educational and research purposes provided that all such whole or partial copies include the following: a notice that such copying is by permission of Mitsubishi Electric Information Technology Center America; an acknowledgment of the authors and individual contributions to the work; and all applicable portions of the copyright notice. Copying, reproduction, or republishing for any other purpose shall require a license with payment of fee to Mitsubishi Electric Information Technology Center America. All rights reserved. 


\section{Publication History:}

1. First printing, TR-2003-38, May 2003 


\title{
Effect of far scatterer clusters in MIMO outdoor channel models
}

\author{
Andreas F. Molisch, Senior Member, IEEE \\ Mitsubishi Electric Research Labs, Murray Hill, NJ, 07974,and \\ Department of Electroscience, Lund University, Lund, Sweden \\ Email: Andreas.Molisch@ieee.org
}

\begin{abstract}
We consider the clustering structure of wireless MIMO channels, and the impact that multiple clusters have on the performance of MIMO systems. We show that multiple clusters frequently occur in urban environments. We then proceed to demonstrate that a channel with multiple scatterer clusters behaves differently than a channel with one cluster and the same rms angular spread. The differences are quantified in terms of correlation coefficients, information-theoretic capacity, and interference-suppression capabilities. This demonstrates the necessity of correctly taking into account the clustering structure.
\end{abstract}

\section{INTRODUCTION}

In recent years, it has been shown that MIMO systems offer very high information-theoretic capacities [1], [2]. This has spurred great interest in such systems; among other applications, they are currently being considered for the high-speed data packet access (HSDPA) of the thirdgeneration standard 3GPP. In order to make realistic evaluations of the capabilities of different system architectures and MIMO schemes, realistic channel models are required.

There are two generic modeling approaches for MIMO channels. So-called "nonphysical models" [3] model the correlation of the fading of the signals at the antenna elements. "Physical models", on the other hand, model the location of scatterers/reflectors, or the direction of multipath components at the transmitter and receiver. Physical models have become more and more popular recently, and are used in such standard models as COST 259 DCM [4] as well as the model developed by the "Spatial Channel Modeling Ad-hoc group" formed by the third-generation standardization organizations 3GPP and 3GPP2 [5].

For physical models, it is a well-established fact that the scatterer locations (or equivalently, the angles and delays of multipath components) are not distributed uniformly over space, but tend to be concentrated in certain regions. The model introduced by [6], which is still widely used today, assumes that all scatterers are located near the mobile station (MS). A more general model, called "multiple cluster" model takes into account not only the scatterers around the MS, but also clusters of "far scatterers" that correspond to high-rise buildings (in urban environments) or mountains (in rural environments) [7],[8].

There is a lot of controversy about the pros and cons of the two approaches, especially in the standardization bodies like 3GPP. It has been argued that (i) multiple clusters do not frequently occur in practice, and (ii) a one-cluster model is equivalent to a "multiple-cluster" model if only the angular spread as seen at the BS and the MS are identical. This is often justified by the work of [9]. In this paper, we will analyze this claim. We will show that while the original work of [9] is clearly correct, it is often misinterpreted, and that identical rms angular spread is not sufficient to guarantee identical MIMO or smart antenna system performance.

The paper is organized the following way: in Section II, we give an overview of the far scatterer model and its physical interpretation. Next, we review some measurement campaigns with respect to the distribution of the angular spectrum. Section IV then discusses the question whether the two approaches are equivalent if only certain measure like the rms angular spread are identical. We will analyze correlation coefficients, MIMO capacity, and interference suppression. A discussion of the solutions in standardized channel models and a summary wrap up this paper.

\section{PHYSICAL INTERPRETATION OF FAR SCATTERER CLUSTERS}

We start out by describing the physical channel modeling with and without far scatterer clusters. The basic form is also known as "local scatterer model" where all relevant scatterers are located around the mobile station (MS). The probability density function (PDF) of the scatterer location is often assumed to be uniform in a disk around the MS, Gaussian, or Rayleigh distributed. Alternatively, the power azimuth delay spectrum shows a single-exponential behavior in delay, and Laplacian behavior in angle, around the (quasi) line-of-sight component [10].

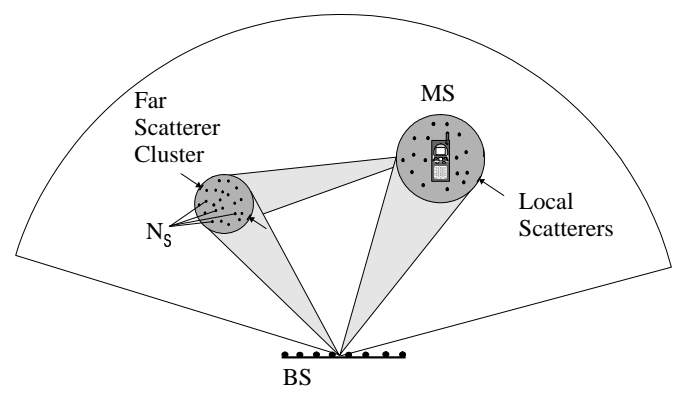

Fig. 1. Principle of far scatterer clusters 
Ref. [7] suggested to additionally model far scatterer clusters that are far away from both the MS and the base station BS (Fig. 1). These far clusters represent e.g. high-rise buildings, in urban environments, mountains in rural environments, etc. While the local scatterers are always centred around the MS, the position of the far scatterers are fixed at an absolute position in space.

Actually, we have to distinguish between two types of far scatterers. One is discrete reflectors, which give rise essentially to a single, specular, multipath component. Such a discrete reflector could be, e.g, a high-rise building with a smooth surface. The other type is a far scatterer cluster, where a group of high rise buildings, or a mountain surface gives rise to several, closely spaced, multipath components. We will see below that this distinction between specular reflectors and scatterer clusters is of special importance for the MIMO capacity.

For microcellular environments, the propagation processes leading to far scatterers are somewhat different. Waves get from the transmitter to the receiver via waveguiding. Different wavesguides thus give rise to different clusters due to different propagation times and/or angles of incidence at the transmitter and receiver. A local cluster, representing over-the-rooftop propagation, is not necessarily present, especially if the BS antenna is significantly below the height of the surrounding rooftops.

The identification of clusters in a measured angular delay power spectrum (ADPS) always involves a certain degree of arbitrariness. We either have to identify an (arbitrary) threshold of the arriving power that the determines the boundary of a cluster, or we have to use "cluster identification algorithms", which are well known from imageprocessing problems., and which give different results according which algorithm is used. In many cases, a visual inspection, together with a knowledge of the morphology of the environments and the possible propagation processes, gives the best results. Naturally, cluster boundaries can be defined better in a channel model.

\section{REVIEW OF MEASUREMENT CAMPAIGNS}

There have been several measurement campaigns that determine the angular spectrum of the signals arriving at the BS:

- Ref. [11] describes a measurement campaign in Frankfurt, Germany. This city has a strong resemblance to many American cities, with a high-rise city center, surrounded by low-rise edifices. The paper clearly identifies many skyscrapers as "far scatterer clusters".

- Ref. [12] paper reports measurements in Paris, France, and is notable in that the directions-of-arrival at the mobile station, but not the BS, are resolved. Also here, several locations are identified where a far scatterer cluster leads to increased delay dispersion. Ref. [13] contains measurements in urban small cells in Mulhouse and Paris, France. The authors observe both cases where there is only a single cluster (with a very small angular spread), as well as cases with several clusters. In one "atypical" case, there was even hardly any energy from the direction of the mobile. However, the case with both a "local" cluster and "far clusters" is judged to be most typical.

- Extensive measurements in Helsinki, Finland [14] have shown that for macrocellular situations (as well as microand picocellular), several clusters are present. 2-3 clusters have to be taken into account to cover $75 \%$ of the energy in $90 \%$ of the cases.

- Ref. [15] report measurements in Stockholm, Sweden. This city is characterized by large areas without buildings (e.g., water), between the built-up areas. Again, multiple clusters have been observed.

Only the measurement campaign of [10] in Bristol, UK, and near Aalborg, Denmark, found the "multiple scatterer cluster" case to be the exception rather than the rule. This can also be explained in terms of the morphology of the measurements; those towns contain fewer irregular structures than the big cities mentioned above.

\section{FAR SCATTERER CLUSTERS IN STANDARDIZED CHANNEL MODELS}

The importance of far scatterer clusters has been recognized also by different standardization bodies. Most notably, the use of far clusters has been recommended (for specific environments) both in the European COST259, which developed a generic spatial channel model that is suitable for a wide range of systems, and by the joint "spatial channel modeling" group of $3 \mathrm{GPP}$ and 3GPP2, the standardization organizations for third-generation cellular systems.

The 3GPP model ${ }^{1}$ defines several environments (suburban macro, urban macro, and urban micro). The suburban macro model uses only a local cluster around the MS, which is in agreement with the measurement results of [10], as described in Sec. III. For the urban macrocell, a single far scatterer cluster (in addition to the local cluster) is an option. It shows the same angular spread as the local cluster, and also exhibits a delay spread. It is thus really a far cluster, and not a discrete scatterer. The urban microcell environment has a total of three clusters, whose mean angle of arrival are independent of the actual location (compare Sec. II). The decision to use far scatterer clusters in 3GPP was partly based on the investigations presented in the current paper.

The incorporation of the far scatterers in the COST259 model is more realistic, and more complicated. The

\footnotetext{
1 This description reflects the status at January 2003, the time of the writing of this paper. A final version of the $3 \mathrm{GPP}$ model is anticipated for March 2003.
} 
number of far scatterer clusters in macrocells is treated as a random variable. Far clusters appear and disappear in a kind of birth/death process, depending on whether a mobile station has line-of-sight to the far scatterer cluster. The mean number of far scatterer clusters depends on the radio environment. For more details, we refer to [16].

\section{IMPACT OF THE NUMBER OF SCATTERER CLUSTERS ON SYSTEM PERFORMANCE}

Having established in Sec. III that far scatterers occur in practice, we now turn to the question whether a far scatterer cluster can be represented by an "equivalent" increase in the rms angular spread of the cluster around the MS.

\section{V.1 Correlation coefficient}

It is well-established [9] that the spatial signature of an antenna array, and thus the correlation coefficient for signals at the elements of a smart antenna, is determined only by the rms angular spread, and not the exact shape of the PAS (power angular spectrum) if the angular spread, and the aperture of the array, is small. This was shown in [9] by using a truncated Taylor expansion of the steering vector. From this point of view, it is thus possible to replace a multiple-cluster model by a single cluster with the same rms angular spread.

However, we have to keep in mind the following restrictions of the derivation (which are also mentioned or implied in [9]):

1. this independence of the shape of the PAS is valid only for the flat-fading case. For the wideband case, multiple clusters give rise to higher frequency selectivity, and also show different angular spectra for different delays. Thus, different types of spatiotemporal Rake receivers work differently in a singlecluster and multiple-cluster scenarios.

2. the independence is only fulfilled if both the rms angular spread and the maximum angular spread are small. Thus, far-off components with small power cannot be represented correctly by increasing the spread of the local cluster. Figure 2 shows the correlation coefficient in the flat-fading case for a single cluster, and for a two-component model (PAS has two delta functions located at 0 and 10,60,120 degrees, respectively) with equal rms angular spread in the flat-fading case.

3. it is required that the antenna pattern is isotropic within the range of occurring angles of incidence. This is especially important when comparing different types of antenna elements, e.g., for different sectorizations of a cell.
4. the independence is true only for complete channel information (CI), where the instantaneous channel characteristics are assumed to be known. This is usually fulfilled on the receiver side. In the FDD case, where the transmitter knows only the average channel characteristics (unless there is a dedicated feedback channel), the most popular approach to exploit the partial channel knowledge is beamforming in the direction of the clusters. This implies that a single-cluster channel exhibits beamforming gain, but not diversity gain. A multicluster scenario offers the possibilities of beamswitching diversity [17], where some parts of a (coded and interleaved) signal are sent into the direction of the local cluster, while other parts arrive at the receiver via far clusters.

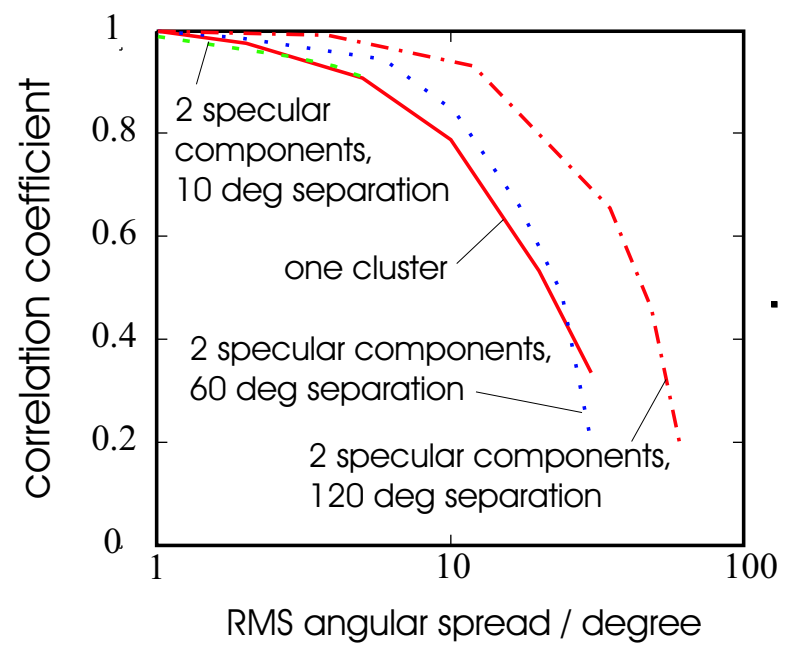

Fig. 2 Correlation coefficient as function of the rms angular spread.

\section{V.2 MIMO capacity}

The channel capacity is the central quantity in the consideration of MIMO systems. We again first present an argument why the capacity should depend only on the angular spread, and then discuss the restrictions of that derivation.

Assume a flat-fading system where scattering processes lead to signal correlations at transmitter and receiver that are independent of each other. In that case, the channel transfer function matrix can be written as

$$
H=R_{R X}{ }^{1 / 2} G R_{T X}{ }^{1 / 2}
$$

where $\mathrm{G}$ is a matrix with i.i.d. complex Gaussian entries, and $\mathrm{R}_{\mathrm{Rx}}$ and $\mathrm{R}_{\mathrm{Tx}}$ are the correlation matrices at transmitter and receiver. If the correlation matrix depends only on the rms angular spread, then this holds also for the matrix $H$, and thus for the capacity 


$$
C=\log _{2}\left(\left|I+\frac{S N R}{N_{t}} H H^{* T}\right|\right)
$$

From this derivation, we can conclude the necessary conditions for the capacity to depend only on the rms angular spread

1. the conditions mentioned in Sec. V.I (flat fading, small rms angular spread, small maximum angular spread) must hold.

2. the channel matrix must allow a decomposition in the form of Eq. (1). This implies that the angular spreading at transmitter and receiver must be independent of each other. A similar condition is also derived in [18] for the limiting case of very large arrays.

3. the considered channel must be a dense multipath channel; i.e., the channel rank must be larger than the number of used antenna elements. If this is not the case, the number of multipath components with large energy (positive SNR) limits the capacity.

4. the computation was done for the capacity of a singlelink MIMO system without co-channel interference. It is not valid in the presence of interference, see Sec. V.3.

We assume in the following that the angular spectrum at the MS is uniform for all delays (this does not correspond to physical reality in the majority of cases, but is sufficient to point out the most essential aspects). We compare now three channel configurations that all have 5.8 (or 30) degree angular spread:

1. a single-cluster model with a Laplacian APS at the BS, with an angular spread of 5.8 and 30 degrees.

2. two equal-power specular sources, one at broadside, and one at 11.6 (or 60) degree angle

3. two scatterer clusters, each with a Laplacian APS with 3 degree angular spread. These clusters have a mean angle-of-arrival of 0 and 10 (or 60) degrees.

Figure 3 shows the cumulative distribution functions of the capacity for $2 * 2,4 * 4$, and $8 * 8$ antenna arrays. We see that for a $2 * 2$ array, the capacity is independent of the shape of the APS, even for the relatively large angular spread of 30 degree. However, for a four-antenna array, this independence is fulfilled only for a small angular spread (5.8 degree), and for an $8 * 8$ array, the capacity shows considerable variations even for a small angular spread.

This behavior of the channel can be explained intuitively. A channel with two specular sources can have at most two significant eigenvalues. Thus, even an increase in the number of antenna elements increases the capacity only by a small amount (by improving the SNR). Note also that for an $8 * 8$ array, the capacities for the two angular spreads coincide almost completely. A single-cluster channel, on the other hand, shows a continuous increase in the effective degrees of freedom (EDF) as the angular spread increases. Since the number of possible propagation paths is much larger, the capacity also exhibits an increase with the number of antenna elements.
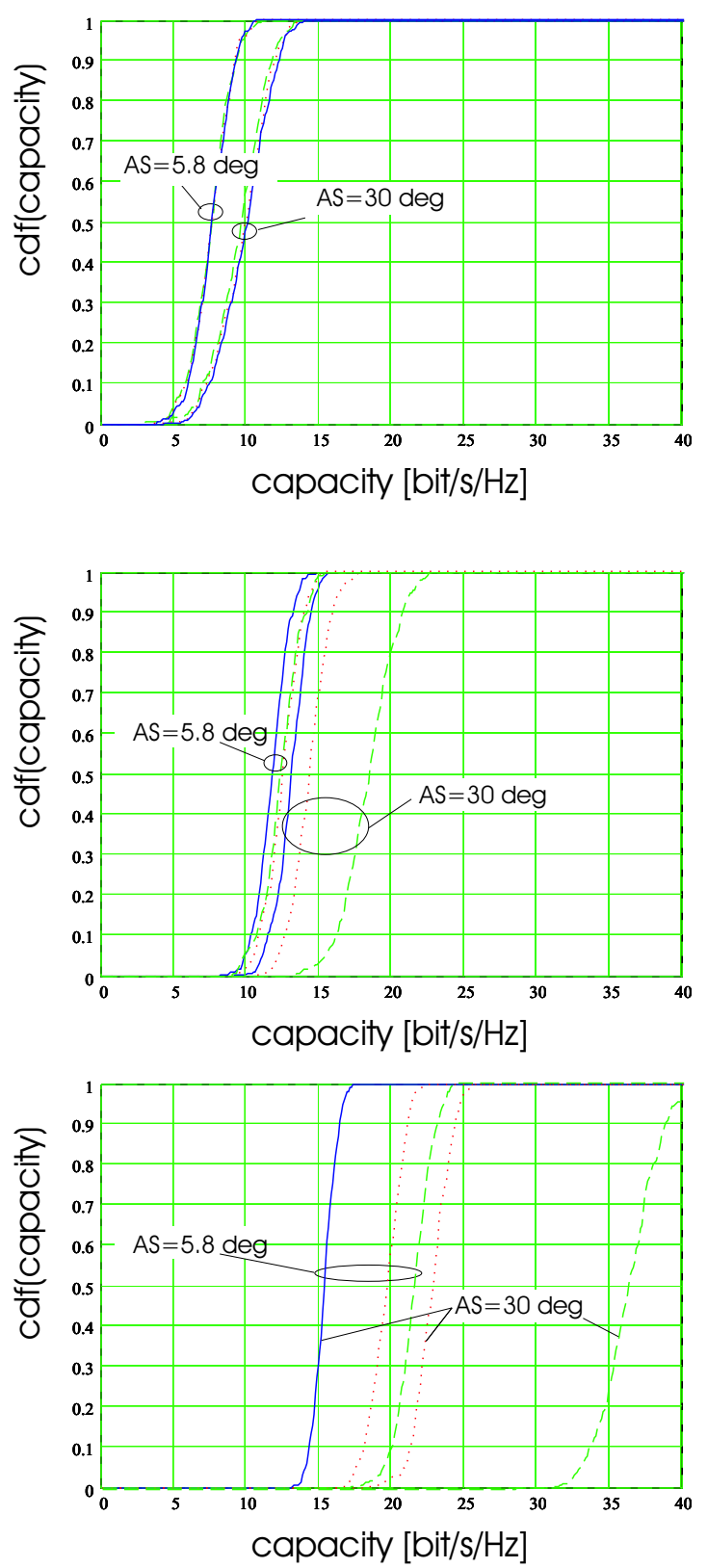

Fig. 3: cdf of the capacity for $2 * 2$ (top), $4 * 4$ (middle) and $8 * 8$ (bottom) antenna arrays and different channels. Two specular sources (solid), single cluster (dashed), two clusters (dotted).

Not surprisingly, a two-cluster channel (with a fixed small but finite angular spread for each cluster) exhibits an intermediate behavior. The capacity does depend on the angular spread, but since the paths within a cluster are so strongly correlated, the effective degree of freedom is mainly determined by the number of clusters, i.e., two. Note also that for large antenna spacing and/or large cluster spread, the capacities of the one-cluster and the two-cluster model become similar (both reduce effectively to an i.i.d. model), 
while the model with two specular paths shows a much smaller capacity determined by the number of significant eigenvalues.

Next consider the capacity as a function of the signal-tonoise ratios for high values of the SNR. An approximate measure for the capacity is the number of eigenvalues of the channel matrix that is larger than the noise variance; we call this number in the following the "effective rank". For the twopath channel, the rank is always two as long as the SNR is larger than the power ratio of the two paths. Increasing the SNR more is not going to change the rank, only the mean SNR. For the one-cluster model, the number of effective eigenvalues is unity as long as the SNR is smaller than the ratio of the first two eigenvalues of the Grammian $\mathrm{HH}^{* \mathrm{~T}}$. However, as we increase the SNR to large values, we will have as many eigenvalues as there are antenna elements.

Another important point is that a two-cluster model shows a significantly larger delay dispersion than a single-cluster model. Simulations (not shown here for space reasons) demonstrate the - not surprising - result that this leads to a steeper slope of the capacity cdf as long as the system bandwidth is larger than the excess delay between the two clusters. For systems with very large bandwidth (where the delay spread within a cluster is comparable to the system bandwidth) that difference vanishes.

Finally, we note that the difference between the twocluster and the single-cluster model can also be explained in the framework of Ref. [19].

\section{V.3 Interference rejection}

Let us consider the downlink, so the problem lies in the suppression of adjacent-cell BS signals. Then the number of data streams that have to be suppressed becomes very large, namely the number of users per cell times the number of antenna elements (for BLAST-like schemes). Thus, an interference suppression based on optimum combining is not feasible; but rather suppression based on directional characteristics has to be used. We now have to distinguish two cases:

- the far scatterer cluster can be a common scatterer for the signal from the desired BS and from the adjacent BS. In that case, it is usually advantageous to apply beamforming, so that the signal from the far cluster, as well as from the direction of the adjacent cell BS location itself, can be suppressed.

- $\quad$ the far scatterer cluster is visible only for the desired BS. In that case, it is advantageous to exploit the signal from the far scatterer.

\section{SUMMARY}

We have demonstrated the importance of far scatterer clusters for the correct modeling of MIMO channels. We have demonstrated that far scatterer clusters frequently occur in urban environments, and that they cannot be replaced by an "equivalent" increase of the angular spread of the scatterer cluster around the MS. Correlation coefficients, MIMO capacity, and interference suppression were shown to behave differently in a wide range of SNRs and array sizes.

Acknowledgement: the author would like to thank the members of the 3GPP3GPP2 ad-hoc group on spatial channel models and Prof. A. Sayeed from Univ. Wisconsin for fruitful discussions.

\section{References}

1 J. H. Winters, "On the capacity of radio communications systems with diversity in Rayleigh fading environments", IEEE J. Sel. Areas Comm., vol. 5, 871-878, 1987.

${ }^{2}$ G. J. Foschini and M. J. Gans, "On Limits of Wireless Communications in a Fading Environment When Using Multiple Antennas", Wireless Personal Communications, vol. 6, 311-335, 1998.

${ }^{3} \mathrm{~K}$. Yu and B. Ottersten, Models for MIMO Propagation Channels, A

Review", Wiley J. Wireless Communications and Mobile Computing, special issue on Smart antenna and MIMO systems, Oct. 2002.

${ }^{4}$ M. Steinbauer and A. F. Molisch, "Directional channel models", Chapter 3.2 in L. Correia (ed.), "Flexible Personalized Wireless Communications", Wiley, 2001.

5 Spatial Channel Model Ad-hoc Group, "Spatial channel model text description v. 2.3", SCM-AHG, 2003.

${ }^{6}$ W. C. Y. Lee, "Effects on correlation between two mobile radio base-station antennas", IEEE Trans. Comm. 21, 1214-1224, 1973.

${ }^{7}$ J. Fuhl, A.F. Molisch, and E. Bonek, "A unified channel model for mobile radio systems with smart antennas", IEE Proc. Radar, Sonar, Navigation, 145, 32-41, 1998

${ }^{8}$ J. Petrus, J. H. Reed, and T. S. Rappaport, "Geometrical-based statistical macrocell channel model for mobile environments", IEEE Trans. Comm., 50, 495-202, 2002.

${ }^{9}$ D. Aszelty and B. Ottersten, "Modified array manifold for signal waveform estimation in wireless communications", $30^{\text {th }}$ Asilomar Conf. Signals, Systems, Comp., pp. 738-741, 1996.

${ }^{10}$ K.I. Pedersen, P.E. Mogensen, B.H. Fleury: Spatial Channel Characteristics in Outdoor Environments and their Impact on BS Antenna System Performance. IEEE Conf. Proc. VTC'98, Ottawa, 1998.

11 U. Martin: Spatio-Temporal Radio Channel Characteristics in Urban Macrocells. IEE Proc. Radar, Sonar and Navigation, vol. 145, pp. 42-49, 1998.

${ }^{12}$ A. Kuchar, J. P. Rossi, and E. Bonek, "Directional macro-cell channel characterization from urban measurements", IEEE Trans. Antennas and Prop., Volume: 48 Issue: 2 , pp. 137-146, 2000.

${ }^{13}$ P. Pajusco: Experimental Characterization of DOA at the Base Station in Rural and Urban Area. Proc. IEEE VTC'98, pp. 993-997, 1998.

${ }^{14}$ M. Töltsch, J. Laurila, A. F. Molisch, K. Kalliola, P. Vainikainen, and E. Bonek, ,Spatial characterization of urban mobile radio channels“, VTC spring 2001, 399-403, 2001.

${ }^{15}$ H. Asplund and J-E. Berg, "Estimation of scatterer locations from urban array channel measurements at $1800 \mathrm{MHz}$," Proc. RadioVetenskap och Kommunikation (RVK'99), pp. 136-140, Karlskrona, Sweden, June 1999.

${ }^{16}$ H. Asplund, A. F. Molisch, M. Steinbauer, and N. Mehta, ,Clustering of scatterers in mobile radio channels - Evaluation and modeling in the COST259 Directional Channel Model", Proc. ICC 2002, 901-905 (2002).

17 A. Kuchar, M. Taferner, M. Tangemann, C. Hoek, W. Rauscher, M. Strasser, G. Pospischil and E. Bonek, "A Robust DOA-based Smart Antenna Processor for GSM Base Stations", ICC'99, pp. 11-16, 1999.

${ }^{18}$ C. N. Chuah, D. N. Tse, J. M. Kahn, R. A. Valenzuela, "Capacity scaling in MIMO wireless systems under correlated fading", IEEE Trans. Information Theory 48, 637-650, 2002.

19 A. Sayeed, "Deconstructing multiantenna fading channels", IEEE Trans. Signal Proc. 50, 2563-2579, 2002. 